\title{
Preliminary Notes
}

PN 1222

\section{Identification of a bacterial phospholipid as an O-ornithine ester of phosphatidyl glycerol}

Recent studies, 1,2 on the phospholipids of Bacillus cereus revealed small amounts of unknown types of phospholipids to be present. During the chromatographic purification of the major phospholipids which resulted in the isolation of pure phosphatidyl glycerol ${ }^{3}$, one of the unidentified minor compounds was also obtained in a chromatographically pure form. Chemical and enzymic hydrolyses carried out on less than I mg of substance allowed this compound to be identified as a derivative of phosphatidyl glycerol esterified with one or more ornithine molecules. The results of this independent study are in fair agreement with those of MACFARLANE ${ }^{4}$ who very recently reported on the occurrence and possible structure of $O$-amino acid esters of phosphatidyl glycerol in Clostridium welchii and Staphylococcus aureus. Indications for the occurrence in small amounts of analogous components in many other bacteria, e.g. Bacillus megaterium, Pseudomonas stutzeri, Serratia marcescens, are accumulating in our laboratory.

After culturing $B$. cereus in a medium containing radioactive orthophosphate, the lipids were extracted and subjected to chromatography on silica columns according to methods outlined previously ${ }^{3}$. Discontinuous elution with mixtures of chloroform and methanol (up to $85: 15, v / v$ ) eluted various acidic phospholipids, phosphatidyl glycerol and phosphatidyl ethanolamine in this order. The compound under discussion was recovered in the fraction chloroform-methanol $(3: 1, v / v)$ sometimes together with a small amount of phosphatidyl glycerol. Rechromatography under identical conditions brought about a separation of the two substances.

The unknown compound showed on silica-impregnated paper chromatograms ${ }^{5}$ and thinlayer chromatograms only one (radioactive) spot, with a $R_{F}$ value resembling that of lecithin. However, the reagent of DRAGENDORFF as used for detection of choline-containing phospholipids ${ }^{6}$ failed to give any positive reaction. The amphiphatic character of the phospholipid was demonstrated by a red colour after treatment with the tricomplex reagent of BUNGENBERG DE JONG ${ }^{7}$. Actually, the phospholipid spot gave a positive ninhydrin reaction. The periodate-Schiff reagent ${ }^{8}$, which is very sensitive for phosphatidyl glycerol, did not give any staining, thus indicating two vicinal hydroxyl groups to be lacking in this new phospholipid.

Mild-alkaline hydrolysis ${ }^{9}$ produced a water-soluble radioactive product, which revealed on two-dimensional paper chromatograms only one radioactive spot coinciding with glycerylphosphoryl glycerol. This finding indicates the close relationship between this phospholipid and phosphatidyl glycerol. Acid hydrolysis gave as expected [ ${ }^{32} \mathrm{P}$ ]glycerophosphate. The hydrolysis product reacting with ninhydrin coincided with both lysine and ornithine on paper chromatograms developed in butanolacetic acid-water $(4: I: 5, v / v)$ and propanol-ammonia-water $(6: 3: 1, v / v)$. However, on paper chromatograms developed in phenol-water, the hydrolysis product behaved 
similarly to ornithine. No indications for the presence of other amino acids or other nitrogenous constituents, e.g. choline, were obtained.

The foregoing observations indicate the isolated phospholipid to be an ornithine derivative of phosphatidyl glycerol (Fig. x). The question can be raised whether this compound contains ornithine esterified to the phospholipid moiety or whether salt linkages between phosphatidyl glycerol and ornithine are involved. Actually LEDERER recovered from mycobacteria ornithine-containing phospholipid fractions, but later he reported this amino acid to be present as an impurity only.10. A liposoluble form of ornithine, which is not a phospholipid, was recently reported by LANÉELLE et al.11.

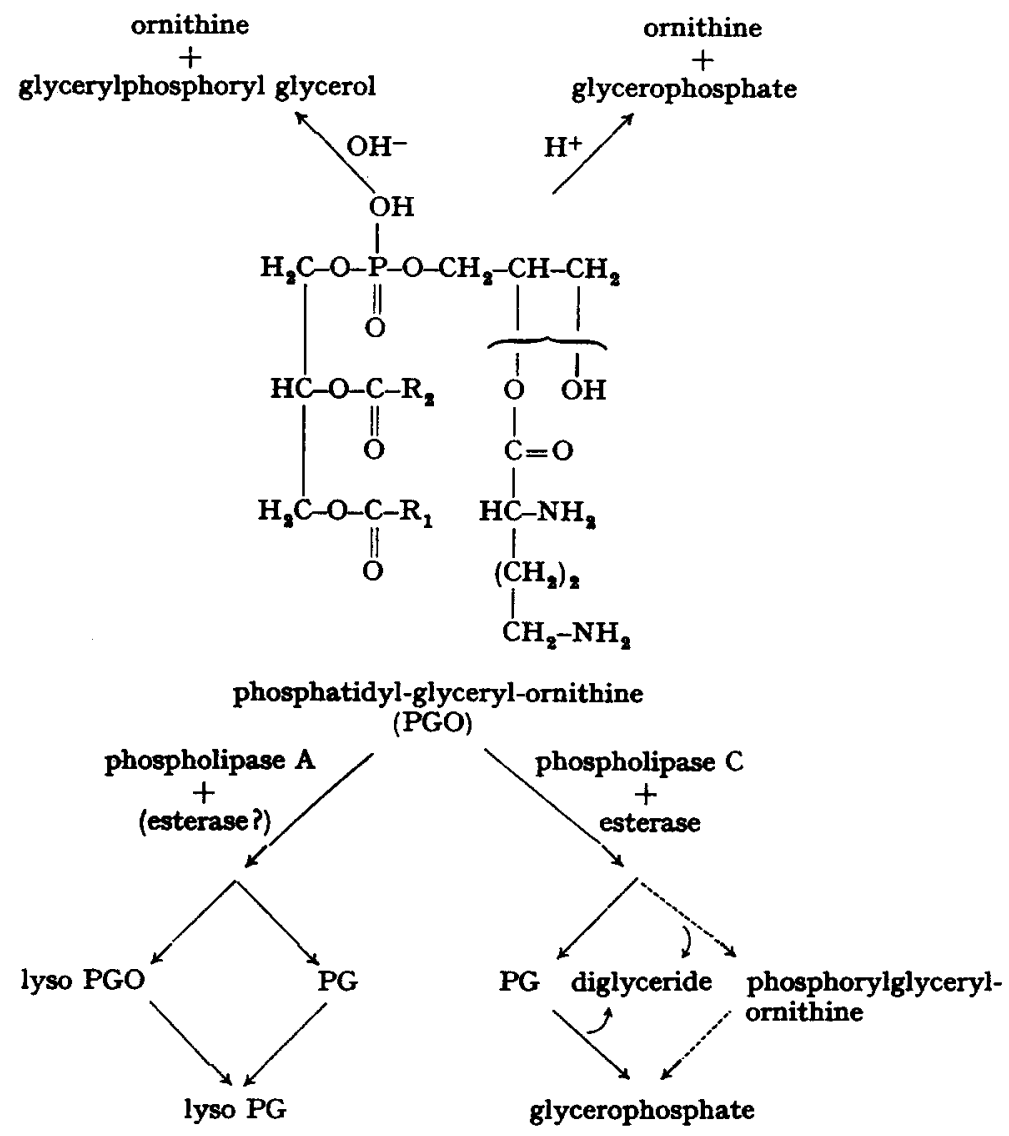

Fig. I. Tentative formula of the isolated phospholipid (PGO) and its breakdown by chemical and enzymic hydrolysis. The pathways indicated by dotted arrows are tentative. $P G=$ phosphatidyl glycerol.

However, in the present study it was shown that phosphatidyl glycerol and ornithine when mixed together under conditions applied for the extraction of the bacterial lipids, did not give rise to the formation of the newly described phospholipid. Paper chromatograms of such mixtures revealed free phosphatidyl glycerol and ornithine, while during paper electrophoresis in an aqueous system ornithine moved also completely free from phosphatidyl glycerol. In contrast, the isolated compound upon 
paper electrophoresis under identical conditions did not give rise to a spot of ornithine, the ninhydrin reaction still coinciding with the phospholipid on the origin. Therefore, it was concluded that in this new phospholipid ornithine is bound to the phosphatidyl glycerol molecule by ester linkage at one or both of the available hydroxyl groups (Fig. I). This view was supported by results obtained by enzymic hydrolysis with phospholipase A (EC 3.I.I.4) and C (EC 3.I.4.3), according to principles applied in a previous investigation on the structure of phosphatidyl glycerol ${ }^{3}$. Phospholipase A (Crotalus adamantheus) produced phosphatidyl glycerol, lysophosphatidyl glycerol and a ninhydrin-positive compound in about the same amount as phosphatidyl glycerol. The $R_{F}$ value of this ninhydrin-positive spot suggested it to be the lyso compound of the ornithine-phospholipid. The appearance of these products may be explained by assuming either the action of an esterase in the snake venom or the ability of phospholipase A to split off amino acids. In the latter case one has to assume one amino acid to be esterified at the $\beta$-position; in the former the absence of more ninhydrin-positive spots would also indicate only one ornithine to be present in the molecule, although an esterase might split off two amino acids at the same time. Phospholipase C, produced by $B$. cereus itself, did cause after a sufficient incubation period a complete hydrolysis of the compound into radioactive glycerophosphate. However, it was observed that during this enzymic incubation also radioactive phosphatidyl glycerol is formed. An esterase is probably present in the crude phospholipase $C$ preparation. It has yet to be settled whether this esterase and phospholipase $C$ are acting simultaneously and/or consecutively.

Although all results obtained so far point to the structure proposed in Fig. I, a more complex molecular arrangement cannot altogether be precluded. MACFARLANE ${ }^{4}$ found in the phospholipid fraction eluted with $20-40 \%$ methanol in chloroform, containing as amino acids mainly ornithine and lysine, a ratio phosphorus-amino nitrogen-total nitrogen of $I: I . I: 2 . I$, showing that only half of the nitrogen is present as amino nitrogen. The amounts of the compound isolated so far by us were not sufficient to ascertain the number and position of the ornithine residues.

This study was supported by the Netherlands Organization for the Advancement of Pure Research (Z.W.O.).

\section{Department of Biochemistry, Laboratory of Organic Chemistry, U. M. T. HoUTSMULLER State University, Utrecht (The Netherlands) $\quad$ L. L. M. VAN DEENEN}

1 M. Kates, D. J. Kushngr and A. T. James, Can. J. Biochem. Physiol., 40 (1962) 83.

${ }^{2}$ L. L. M. VAn Deenen and U. M. T. Houtsmulere, Proc. Deuel Conf. on Lipids, Santa Barbara, February, $x 962$, in the press.

F. Haverkate, U. M. T. Houtsmuller and L. L. M. Van Deenen, Biochim. Biophys. Acta, $63(\mathrm{I} 962) 547$.

M. G. Machariant, Nature, 196 (I962) 136.

5 G. V. Marinetti, J. Erbland and J. Kochen, Federation Proc., 16 (1957) 837.

- F. Kögl and L. L. M. Van Deenen, Acta Endocrinol., 36 (196r) 9.

7 H. G. Bungenberg de Jong, Koninkl. Ned. Akad. Wetenschap. Proc. Ser. B., 64 (I961) 583.

8 J. Baddirty, J. G. Buchanan, R. E. Handschummacher and J. F. Prescott, J. Chem. Soc., (I956) 28 r8.

A. A. Benson and B. Maruo, Biochim. Biophys. Acta, 27 (1958) I89.

10 E. LeDRRER, Biochem. $J$, 8I (I96I) 3I P.

11 M. A. Lantelle, G. Lantelle and J. Asselineau, Biochim. Biophys. Acta, 70 (1963) 99.

Received December 2oth, I962 